\title{
Estimating nest abundance while accounting for time-to-event processes and imperfect detection
}

\author{
Guillaume Péron, ${ }^{1,4,5}$ Johann Walker, ${ }^{2}$ Jay Rotella, ${ }^{3}$ James E. Hines,${ }^{4}$ and James D. Nichols ${ }^{4}$ \\ ${ }^{1}$ Colorado Cooperative Fish and Wildife Research Unit, Department of Fish, Wildlife, and Conservation Biology, \\ 1484 Campus Delivery, Colorado State University, Fort Collins, Colorado 80523-1484 USA \\ ${ }^{2}$ Ducks Unlimited, Inc., Great Plains Regional Office, 2525 River Road, Bismarck, North Dakota 58503 USA \\ ${ }^{3}$ Department of Ecology, Montana State University, Bozeman, Montana 59717 USA \\ ${ }^{4}$ Patuxent Wildife Research Center, U.S. Geological Survey, 12100 Beech Forest Road, Laurel, Maryland 20708-4017 USA
}

\begin{abstract}
Birds and their population dynamics are often used to understand and document anthropogenic effects on biodiversity. Nest success is a critical component of the breeding output of birds in different environments; but to obtain the complete picture of how bird populations respond to perturbations, we also need an estimate of nest abundance or density. The problem is that raw counts generally underestimate actual nest numbers because detection is imperfect and because some nests may fail or fledge before being subjected to detection efforts. Here we develop a state-space superpopulation capture-recapture approach in which inference about detection probability is based on the age at first detection, as opposed to the sequence of re-detections in standard capture-recapture models. We apply the method to ducks in which (1) the age of the nests and their initiation dates can be determined upon detection and (2) the duration of the different stages of the breeding cycle is a priori known. We fit three model variants with or without assumptions about the phenology of nest initiation dates, and use simulations to evaluate the performance of the approach in challenging situations. In an application to Blue-winged Teal Anas discors breeding at study sites in North and South Dakota, USA, nesting stage (egg-laying or incubation) markedly influenced nest survival and detection probabilities. Two individual covariates, one binary covariate (presence of grazing cattle at the nest site), and one continuous covariate (Robel index of vegetation), had only weak effects. We estimated that $5-10 \%$ of the total number of nests were available for detection but were missed by field crews. An additional 6-15\% were never available for detection. These percentages are expected to be larger in less intense, more typical sampling designs. User-friendly software nestAbund is provided to assist users in implementing the method.
\end{abstract}

Key words: Anas discors; Blue-winged Teal; capture-recapture; habitat choice; nest success; North and South Dakota, USA; population density; population dynamics; Prairie Pothole Region.

\section{INTRODUCTION}

Birds and their population dynamics are often used to understand and document anthropogenic effects on biodiversity, such as habitat fragmentation (Newmark and Stanley 2011) and climate change (Jiguet et al. 2010), but also a priori benign activities such as nature tourism (Kerbiriou et al. 2009). Nest success is often used to measure the effect of perturbations, and it has been the subject of a rich literature (Mayfield 1961, Ricklefs 1969, Pollock and Cornelius 1988, Martin 1995, Dinsmore et al. 2002, Rotella et al. 2004, Jones and Geupel 2007, Newmark and Stanley 2011). Nest success, however, is only one component of bird breeding productivity. Total nest abundance, or breeding density, may also vary in time and space. For example,

Manuscript received 20 September 2012; revised 5 March 2014; accepted 7 March 2014. Corresponding Editor: E. G. Cooch.

${ }^{5}$ E-mail: peron_guillaume@yahoo.fr individuals may try to avoid areas where their reproductive success would be lower than average (Péron et al. 2010), or, in contrast, they may fall victim to ecological traps when anthropogenic change alters habitat quality but not the habitat cues that the birds use (Robertson and Hutto 2006). However, estimating total nest abundance requires that two potential methodological pitfalls are addressed. First comes the problem of imperfect detection, that is, observers in the field generally cannot detect all of the nests that are available at the time of surveys. Second, nests are subject to time-to-event processes (Kalbfleisch and Prentice 2002, Collett 2003): eggs eventually hatch and nestlings fledge, and nests also risk depredations and nest abandonment. In other words, nests have a finite life span during which they are available for detection. As a result, the count of detected nests generally underestimates the total number of nests in an area. The same problem also exists for other immobile or sessile "objects" in nature, such as plants, benthic invertebrates, dung piles, and territorial animals. 
Here we propose to use capture-recapture models, and in particular "superpopulation" models (Crosbie and Manly 1985, Schwarz and Arnason 1996), to address those questions and correct raw counts of detected nests for imperfect detection and finite life span (Williams et al. 2002). First we develop a general framework presenting the challenges associated with inference about nests and other immobile or stationary objects in nature. Then we present a specific case of the model tailored for Anatidae bird species. We analyze a data set from Blue-winged Teal Anas discors at study sites in the Prairie region of North and South Dakota, USA. The number of offspring produced each season by a unit of habitat (or a population) is of critical importance to assess habitat management efforts, to project population dynamics, and to study habitat choice in an evolutionary context. Finally, we review methodological bottlenecks remaining after our work, as well as model features that could readily be included in future model extensions. The analyses in this article can be reproduced using R-package nestAbund, which has a user-friendly interface (available online). ${ }^{6}$

\section{General Framework: Time-to-Event Processes and Bird Nests}

The different stages of the nesting season (egg-laying, incubation, chick-rearing) are well known; and the biological process by which a nest passes through these three stages may appear trivial (Fig. 1). But, when combined with a mortality risk (depredation and nest abandonment), and with imperfect detection, this process produces a large number of possible observations. A nest may be detected during any of the three stages and may fail at any point of the cycle, which is sometimes referred to as interval censoring (Stanley and Newmark 2010). For each nest we define $D_{1}$ as the date at nest initiation (first egg laid); $D_{2}$ as the date at clutch completion and start of incubation; $D_{3}$ as the hatching date; and $D_{4}$ as the fledging date. These dates are linked by the nest-specific stage durations: $L_{1}$, the length of the egg-laying period; $L_{2}$, the length of the incubation period; and $L_{3}$, the length of the chick-rearing period. A last variable of interest is $E$, the initial clutch size at the start of incubation. Data collected from each nest are $f$, the date when the nest was first detected; $z$, the date it was last observed before either fledging or failing; $s$, which equals 1 if the nest succeeded and zero otherwise; and $E$, the clutch size (if available; not recorded if the nest failed before clutch completion). In the following, to simplify the presentation at this stage, we assume that (1) the study area is visited daily; (2) detected nests are monitored until their fate is recorded; (3) there is a deterministic relationship between clutch size and duration of the egg-laying period: $L_{1}=\alpha E$, where $\alpha$ is

\footnotetext{
${ }^{6}$ http://www.mbr-pwrc.usgs.gov/software/nestAbund.html
}

the number of eggs laid per day and is considered known ( $\alpha=1$ in many species including Blue-winged Teal); and (4) the duration of the incubation period is the same for all nests: $L_{2}$ is a constant. In addition to these, the model may require assumptions about the parametric form of the distributions for the duration of the chick-rearing period $L_{3}$, nest initiation date $D_{1}$, and clutch size $E$. Model parameters and notation are summarized in Table 1.

Let's then first express the probability of nest success (probability that a nest survives to produce at least one fledgling) for a nest with known values of the covariates $D_{1}, L_{1}, L_{2}, L_{3}$ :

$$
\operatorname{Pr}\left(s=1 \mid D_{1}, L_{1}, L_{2}, L_{3}\right)=\prod_{i=D_{1}}^{D_{2}-1} \varphi_{i}^{(1)} \prod_{i=D_{2}}^{D_{3}-1} \varphi_{i}^{(2)} \prod_{i=D_{3}}^{D_{4}-1} \varphi_{i}^{(3)}
$$

Second, let $\operatorname{Pr}\left(z \mid D_{1}, L_{1}, L_{2}, L_{3}, s=0\right)$ be the probability that the same nest fails on date $z$ :

$$
\begin{aligned}
& \operatorname{Pr}\left(z \mid D_{1}, L_{1}, L_{2}, L_{3}, s=0\right)= \\
& \left\{\begin{aligned}
{\left[\prod_{i=D_{1}}^{z-1} \varphi_{i}^{(1)}\right]\left(1-\varphi_{z}^{(1)}\right), } & \text { if } \quad D_{1}<z<D_{2}
\end{aligned}\right. \\
& {\left[\prod_{i=D_{1}}^{D_{2}-1} \varphi_{i}^{(1)}\right]\left[\prod_{i=D_{2}}^{z-1} \varphi_{i}^{(2)}\right]\left(1-\varphi_{z}^{(2)}\right), \quad \text { if } \quad D_{2} \leq z<D_{3} .} \\
& {\left[\prod_{i=D_{1}}^{D_{2}-1} \varphi_{i}^{(1)}\right]\left[\prod_{i=D_{2}}^{D_{3}-1} \varphi_{i}^{(2)}\right]\left[\prod_{i=D_{3}}^{z-1} \varphi_{i}^{(3)}\right]\left(1-\varphi_{z}^{(3)}\right), \quad \text { if } \quad D_{3} \leq z<D_{4}}
\end{aligned}
$$

Note that if failure occurs before the clutch is completed, then clutch size $E$ is not recorded but $L_{1}$ does not enter the formula so information about $E$ is not required. Third, let $\operatorname{Pr}\left(f \mid D_{1}, L_{1}, L_{2}, L_{3}\right)$ be the probability that the nest is detected on day $f$ but not earlier, conditional on survival until day $f$ :

$$
\begin{aligned}
& \operatorname{Pr}\left(f \mid D_{1}, L_{1}, L_{2}, L_{3}\right)=
\end{aligned}
$$

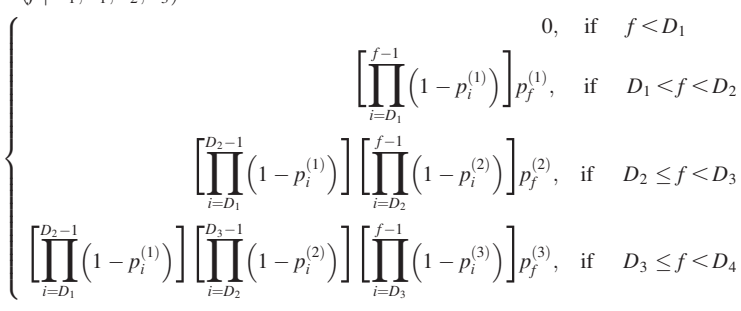

Note that Eq. 3 deviates from the standard way of modeling detection history data in superpopulation capture-recapture models. The usual assumption for mobile organisms is that initial and subsequent detec- 


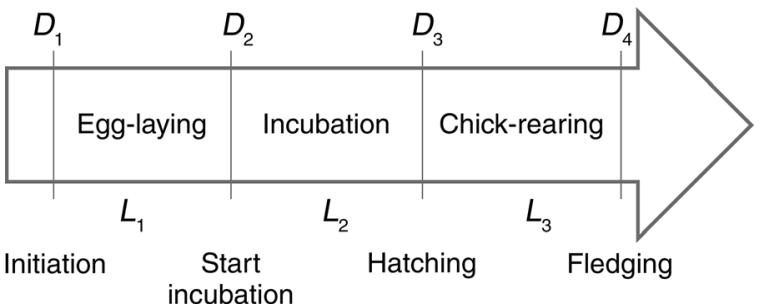

FIG. 1. Generalized breeding cycle with notation used in this article: $D_{1}, D_{2}, D_{3}$, and $D_{4}$ are, respectively, the nest initiation date, the date at the start of incubation, hatching date, and fledging date. They are separated by the egg-laying period lasting $L_{1}$ days, the incubation period lasting $L_{2}$ days, and the chick-rearing period lasting $L_{3}$ days.

tions are governed by the same probabilities, such that detection history data collected after the initial encounter are adequate for inference about detection. However, following initial detections, locations of immobile objects such as nests are known, so that subsequent detections are near-certain and provide little or no information about initial detection probability. Inference about detection probability is here made conditional on $D_{1}$, so that we know the number of times the nest escaped detection.

Eqs. 1 and 2 describe the state process of transition between stages and nest failure or success. Eq. 3 describes the observation process. These processes are linked to the data as follows for the $k$ th detected nest:

$$
\begin{aligned}
\operatorname{Pr}\left(f_{k}, z_{k}, s_{k}, E_{k}\right)= & \\
\operatorname{Pr}\left(E_{k}\right) \int_{D_{1}} \int_{L_{3}}[ & \\
& s_{k} \operatorname{Pr}\left(s=1 \mid D_{1}, \alpha E_{k}, L_{2}, L_{3}\right) \\
& \left.+\left(1-s_{k}\right) \operatorname{Pr}\left(z_{k} \mid D_{1}, \alpha E_{k}, L_{2}, L_{3}, s=0\right)\right] \\
& \left.\times \operatorname{Pr}\left(f_{k} \mid D_{1}, \alpha E_{k}, L_{2}, L_{3}\right)\right] \operatorname{Pr}\left(L_{3}\right) \operatorname{Pr}\left(D_{1}\right) \\
& \times d L_{3} d D_{1}
\end{aligned}
$$

where $\operatorname{Pr}(E), \operatorname{Pr}\left(L_{3}\right)$, and $\operatorname{Pr}\left(D_{1}\right)$ denote the distribution among nests of clutch size, chick rearing period, and initiation date, respectively. Then, we compute the probability of not finding a nest that is in the superpopulation, denoted $P_{0}$. Let's first define the probability of not finding a nest that is initiated on day $D_{1}$ and is available for detection until day $z\left(z>D_{1}\right)$ :

$$
\begin{aligned}
& \operatorname{Pr}\left(\text { "not found" } \mid \mathrm{z}, \mathrm{D}_{1}, \mathrm{~L}_{1}, \mathrm{~L}_{2}, \mathrm{~L}_{3}\right)= \\
& \left\{\begin{aligned}
& {\left[\prod_{i=D_{1}}^{z-1}\left(1-p_{i}^{(1)}\right)\right], } \text { if } \quad D_{1}<z<D_{2} \\
& {\left[\prod_{i=D_{1}}^{D_{2}-1}\left(1-p_{i}^{(1)}\right)\right]\left[\prod_{i=D_{2}}^{z-1}\left(1-p_{i}^{(2)}\right)\right], } \text { if } \quad D_{2} \leq z<D_{3} . \\
& {\left[\prod_{i=D_{1}}^{D_{2}-1}\left(1-p_{i}^{(1)}\right)\right]\left[\prod_{i=D_{2}}^{D_{3}-1}\left(1-p_{i}^{(2)}\right)\right]\left[\prod_{i=D_{3}}^{z-1}\left(1-p_{i}^{(3)}\right)\right], \quad \text { if } \quad D_{3} \leq z<D_{4} }
\end{aligned}\right.
\end{aligned}
$$

Then the probability of missing a nest for which we do not know the values of $z, D_{1}, L_{1}, L_{3}$ is:

$$
\begin{aligned}
P_{0}= & \int_{E} \int_{D_{1}} \int_{L_{3}}\left[\operatorname{Pr}\left(s=1 \mid D_{1}, \alpha E, L_{2}, L_{3}\right)\right. \\
\times & \left.\operatorname{Pr} \text { ("not found" } \mid \mathrm{D}_{1}+\alpha \mathrm{E}+\mathrm{L}_{2}+\mathrm{L}_{3}, \mathrm{D}_{1}, \alpha \mathrm{E}, \mathrm{L}_{2}, \mathrm{~L}_{3}\right) \\
& +\sum_{z=D_{1}+1}^{D_{4}-1} \operatorname{Pr}\left(z \mid D_{1}, \alpha E, L_{2}, L_{3}, s=0\right) \\
& \left.\times \operatorname{Pr}\left(\text { "not found" } \mid z, D_{1}, L_{1}, \alpha E, L_{3}\right)\right] \\
& \times \operatorname{Pr}\left(L_{3}\right) \operatorname{Pr}\left(D_{1}\right) \operatorname{Pr}(E) d E d L_{3} d D_{1} .
\end{aligned}
$$

Finally, denoting $\boldsymbol{\Theta}$ the vector containing all model parameters, the likelihood of the complete data set is obtained by summing across all $C$ observed nests and $N$ - $C$ non-observed nests (Schwarz and Arnason 1996):

$$
\begin{aligned}
& L\left(\Theta \mid\left\{f_{k}, z_{k}, s_{k}, E_{k}\right\}_{k=1 \ldots C}\right) \propto \\
& \quad\left[\prod_{k=1}^{C} \operatorname{Pr}\left(f_{k}, z_{k}, s_{k}, E_{k}\right)\right] \frac{N !}{(N-C) !} P_{0}^{N-C} .
\end{aligned}
$$

\section{Realistic Model Variants}

In this section we describe modifications brought to the previously described general model to make it relevant for real-life data sets. Further potential modifications and extensions are tackled in the discussion. First, the study period does not compulsorily encompass a representative section of the breeding season; thus, only part of the distribution of $D_{1}$ is represented in the data. This is readily accommodated by restricting the inference to the superpopulation of nests that are available for detection during at least one site visit (Schwarz and Arnason 1996). In this case, instead of modeling the distribution for $D_{1}$, we estimate entry probabilities: these represent for each time interval the probability that a nest of the superpopulation is initiated during this interval. Second, to reduce disturbance to nesting birds, most sites are typically visited not on a daily basis, but rather at intervals of several days. We used interval survival rates rather than daily survival. Last, the homogeneity assumption (the as-

TABLE 1. Model parameters.

\begin{tabular}{cc}
\hline \hline Notation & \multicolumn{1}{c}{ Biological meaning } \\
\hline$N$ & $\begin{array}{c}\text { Superpopulation size. In the most general models this } \\
\text { includes all nests initiated in the study area. In } \\
\text { restricted applications (staggered-entry model) this } \\
\text { represents nests that are available for detection }\end{array}$ \\
& during at least one sampling occasion. It includes \\
& renesting attempts after the failure of initial \\
& attempts and does not discriminate between those \\
& and initial attempts. \\
$\varphi_{i}^{(s)}$ & Daily nest survival for day $i$ in state $s$. \\
$p_{i}^{(s)}$ & Detection probability for day $i$ in state $s$. \\
$\delta$ & Expected initiation date. \\
$\sigma$ & Standard deviation of initiation date. \\
\hline
\end{tabular}


sumption that detected nests are representative of undetected nests) might not be verified. In this study, this issue is partly addressed through the use of individual covariates, i.e., nest-specific variables that are believed to impact on nest fate (survival and/or detection). For example, nest success probability may vary with the local predator assemblage and initiation date (Calero-Riestra et al. 2013). In superpopulation models, this involves summing over all possible nestspecific covariate values in order to estimate $P_{0}$, the probability of not finding a nest with unknown covariates. The use of covariates also allows one to control for potential covariation between nest success and nest detection probability (e.g., via variation in nest concealment).

A last set of modifications was brought to tailor the model to Anatidae, and Blue-winged Teal, in particular. Anatidae constitute an ideal group of species for applying our method for the first time because several features allow simplification of the model. First, by using the candling method, we know when the detected nests were initiated $\left(D_{1}\right)$. Although the candling method can be imprecise, for example if the eggs are stained, we assume here as a first approximation, and following most previous works on duck breeding success, that there is no error. We can then compute exactly how many times a nest went undetected (Eq. 3). Second, ducks have precocial young that leave the nest within hours of hatching, so we can fix $L_{3}=0$, i.e., nest success is measured by hatching success. Third, incubation length $L_{2}$ is known and does not need to be estimated ( $L_{2}=24$ days in Blue-winged Teal). Finally, when computing $P_{0}$ (Eq. 6) we did not consider all possible values of $E$, but rather fixed it to the modal value $(E=10$ eggs), so that the length of the egg-laying period was fixed to $L_{1}=10 \mathrm{~d}$.

\section{Application Case \\ Field procedures and data description}

Our application case is based on data collected in 2012; we collected nest observations on four plots owned by Ducks Unlimited in the Prairie Pothole Region of North and South Dakota. Plot centroids were located at $100.261 \mathrm{~W}, 47.418 \mathrm{~N} ; 100.262 \mathrm{~W}$, $47.396 \mathrm{~N}$; $99.148 \mathrm{~W}, 45.597 \mathrm{~N}$; and $99.163 \mathrm{~W}, 45.596$ $\mathrm{N}$, and plot areas were 56, 59, 90, and 88 ha, respectively. Land cover of the plots was dominated by herbaceous perennial vegetation. Two of the plots were grazed by cattle during part of the nesting season. We searched the study plots for nests at approximately weekly intervals (range 6-9 d) using all-terrain vehicles and chain-drags (Klett et al. 1986; see Plate 1). When we found a nest, we recorded date, species, and nest age. Nest age was determined by candling 2-3 eggs from each nest to assess their developmental stage (Weller 1956, Ackerman and Eagles-Smith 2010). To describe variation in nest concealment, we recorded Robel's index of vegetation at the nest site (Robel et al.
1970). We marked each nest with a uniquely numbered $100 \times 1 \mathrm{~cm}$ fiberglass rod placed $5 \mathrm{~m}$ north of the nest site, and we recorded the geographic coordinates of the nest with a handheld GPS receiver. We revisited previously discovered nests at intervals of 2-7 days until the eggs hatched or the nest failed. In total, 401 duck nests were detected $(44,61,153$, and 143 in each of the four sites). Of these, 207 (31, 35, 71, 70) were Blue-winged Teal nests. Teal nests, on average, were initiated $8.5 \mathrm{~d}$ before they were detected $(\mathrm{SD}=4.5 \mathrm{~d})$, and overall $6.2 \mathrm{~d}$ after the first day of the study ( $\mathrm{SD}=$ 11.9 d). The average Robel index of vegetation at each nest was $1.73(\mathrm{SD}=0.63)$; 90 nests had cattle grazing on the field they were located in (sites 2 and 4 only). In this study, we focused on the following effects acting on nest success and detection probability: site, Robel index of vegetation, presence of grazing cattle, and nesting stage. Female ducks spend less time attending their nest during the egg-laying period; thus, a nest might be less likely to both be depredated and detected by humans during the egg-laying period than during the incubation period.

\section{Specificities of the present data set}

Our data are exceptional in some ways compared to other nest-searching data. First, the study was designed to encompass most of the breeding season at each of the four sites, so that we could safely assume that almost the whole range of nest initiation dates was sampled and only the tails of the distributions were lacking. Second, we knew the fate of all of the detected nests (no right-censoring). Third, the field effort (number of site visits, detection probability during each visit, and interval between them) was intensive enough that we were confident that $P_{0}$ was small. We also observed that the distribution of initiation dates among detected nests was significantly platykurtic (with "broad shoulders" and few very early and late nests; Anscombe-Glynn kurtosis test: kurtosis $=2.40, P$ $=0.02$; Fig. 4 ). This could be expected due to the fitness benefits of nesting early and the fact that hens generally renest after failure. There was no skewness (d'Agostino test: skew $=-0.35 ; P=0.17)$. These elements suggested that the raised cosine distribution, with a kurtosis value of $\sim 2.41$ (close to the 2.40 observed value), was more adapted to this specific data set than the Gaussian distribution. This distribution has two parameters akin to mean and standard deviation that we included in the parameter set.

\section{Data analysis}

As previously introduced, we fit three variants of superpopulation models that differed in the way the temporal distribution of nest initiation dates was modeled. First, we fit a "standard" superpopulation model in which a set of entry probability parameters represented the temporal distribution. In this model, we estimated the number of nests available for detection 

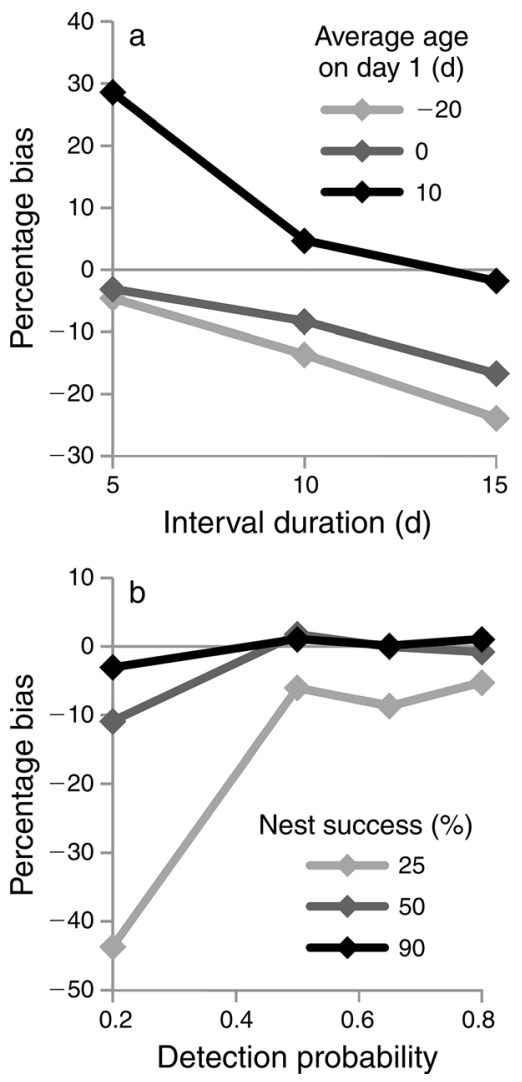

FIG. 2. Simulation results from 148 data sets. (a) Variation in percentage bias in superpopulation size estimate with interval duration between two visits to the study sites, when the study starts on three different dates. "Average age on day 1" represents the difference in days between peak initiation date and start of the study (negative if the peak date is after the start of the study). (b) Variation in percentage bias in superpopulation size estimate with nest detection probability, for three levels of nest success. Bias is the percentage difference between true and estimated superpopulation size. The standard deviation of initiation date was always 12 days. Each point corresponds to an average across 6-9 scenarios depending on the occurrence of numerical instabilities (see Results: Simulation study).

during at least one site visit. These nests were thus initiated within a specific time frame determined by the first and last visits to the sites, and they survived at least until the next site visit. Second, we fit the model in which nest initiation date was normally distributed. For this model we used the Gauss-Hermite quadrature to compute $P_{0}$ (Golub and Welsch 1969, Gimenez and Choquet 2010). Third, we fit the model in which nest initiation date followed the raised cosine distribution. For this we used a Riemann sum approximation to compute $P_{0}$; i.e., we assigned a $P_{0}$ value to each possible $24-\mathrm{h}$ interval and then summed over $24-\mathrm{h}$ intervals. In the latter two models, the estimated superpopulation size includes all nests, even those that were initiated after the study period or that died before being subjected to the detection process. This was made possible by the strong assumption about temporal distribution. Note that we did not fit a site-specific distribution, but used the same distribution for all four sites. We did not use the Akaike information criterion to decide upon a preferred model variant, because the estimated quantities were actually not the same, and because of the technical differences in the way the likelihood was computed (different approximations). To fit models with the effect of the binary individual covariate "presence of cattle," we introduced a site-specific parameter "proportion of nest sites that have grazing cattle." To fit models with the effect of the continuous covariate "Robel index," we assumed that Robel index was normally distributed among nests and used the GaussHermite quadrature to compute $P_{0}$. Statistical significance of the biological effects (site, nesting stage, Robel index, and presence of cattle) was determined using the $95 \%$ confidence interval ( $95 \%$ CI) obtained by inversing the Hessian of the log-likelihood function at its minimum. If the $95 \% \mathrm{CI}$ included zero (or overlapped in the case of site effects), we considered the effect as nonsignificant. We also ran a parametric bootstrap (250 replications) to compute $95 \%$ CI on transformed estimates and derived quantities. Simpler models required a few minutes to converge, but the full model with both covariates and staggered entry could require up to several hours on some desktop computers. The variant with Gaussian distribution was the fastest to run. We somewhat reduced computation time by first fitting a closed-population capture-recapture model and then using its estimate as the starting value in the optimization routine for the superpopulation model. We provide user-friendly software nestAbund as an Rpackage to reproduce our analyses (see footnote 6).

\section{Simulations}

We generated and analyzed data for 108 scenarios, mostly with the aim of illustrating problematic situations pertaining to our new developments, not to superpopulation capture-recapture models in general. Across scenarios, nest success (percentage hatching) could be $25 \%, 50 \%$, or $90 \%$ (corresponding to daily nest survival of $3.2,3.9,5.8$ on the logit scale); detection probability could be $0.2,0.5$, or 0.8 ; mean initiation date could be 10 days before the start of the study, at the start of the study, or 20 days after it; the interval between successive sampling occasions could be 5,10 , or 15 days. Note that an interval duration of 15 days meant that nests had at most two chances of being detected before fledging/failing. In all scenarios, $L_{1}$ and $L_{2}$ were fixed to the values for Blue-winged Teal. Standard deviation of initiation date was always 20 days and the distribution was Gaussian. The study ended on day 100 , largely after the last nest fledged. We simulated a total superpopulation of 1000 nests. We analyzed these data using the Gaussian model only and computed the bias (percentage difference between true and estimated superpopulation size) for the various scenarios. We fit 
TABLE 2. Estimates for the model including all considered effects acting on survival and detection probabilities of Blue-winged Teal Anas discors nests in North and South Dakota (USA) study sites, with $95 \%$ confidence intervals (lower 2.5th and upper 97.5th quantiles).

\begin{tabular}{lrrr}
\hline \hline \multicolumn{1}{c}{ Parameter } & Estimate & 2.5th quantile & 97.5 th quantile \\
\hline Survival & & & $\mathbf{5 . 9 0 4}$ \\
Site 1 & $\mathbf{4 . 8 8 1}$ & $\mathbf{3 . 8 5 9}$ & 3.725 \\
Site 2 & 2.947 & 2.170 & 3.948 \\
Site 3 & 3.409 & 2.871 & 3.412 \\
Site 4 & 2.626 & 1.840 & $\mathbf{- 0 . 1 3 5}$ \\
Egg-laying vs. incubation & $-\mathbf{0 . 4 9 8}$ & $\mathbf{- 0 . 8 6 1}$ & $\mathbf{1 . 5 6 5}$ \\
Cattle & $\mathbf{0 . 9 0 6}$ & $\mathbf{0 . 2 4 8}$ & 0.179 \\
Robel & -0.097 & -0.373 & 2.344 \\
Detection & & & 4.559 \\
Site 1 & 1.134 & -0.076 & 2.436 \\
Site 2 & 2.848 & 1.137 & 4.199 \\
Site 3 & 1.558 & 0.681 & $-\mathbf{1 . 0 0 7}$ \\
Site 4 & 2.491 & 0.782 & 0.209 \\
Incubation vs. egg-laying & $-\mathbf{1 . 5 7 9}$ & $-\mathbf{2 . 1 5 1}$ & 0.201 \\
Cattle & -1.296 & -2.801 & -0.524 \\
Robel & -0.162 & & \\
\hline
\end{tabular}

Notes: These estimates correspond to the parameters from logit-linear regressions of survival and detection probabilities against site identity (site 1 to 4 ), nesting stage (egg-laying or incubation), presence of cattle near the nest, and Robel index of vegetation density near the nest. Significant parameters (as per the $95 \%$ confidence intervals) are indicated in bold.

linear models in order to identify the study design variables that led to largest bias.

\section{RESUlts}

\section{Simulation study}

First, we observed numerical instabilities $\left(P_{0}\right.$ estimated near 1.0 and population size estimate approaching infinity) for all scenarios with both low detection probability $(0.2)$ and long intervals between site visits (10 or $15 \mathrm{~d}$ ); these scenarios were excluded from further analysis and we recommend not using the method with such data. Negative bias on superpopulation size estimate increased with interval duration $(-1.70 \% \pm$ $0.40 \% \mathrm{bias} / \mathrm{d}$, estimate $\pm \mathrm{SE}$; ANOVA $P<0.001$; Fig. 2a) and with time difference between the start of the study and peak nest initiation date $(-0.78 \% \pm$ SE $0.13 \%$ bias/d; $P<0.001$; Fig. 2a). Low values of survival probability and of detection probability also led to larger biases $(P<0.001$; Fig. 2 b). We thus observed as expected that (1) scenarios with long intervals between nest searches led to underestimating superpopulation size because detected nests were a nonrepresentative sample and (2) when nest success or detection probability were low, this bias was amplified. In favorable scenarios (nest success $50 \%$ or above, detection probability $50 \%$ or above, interval duration of 5 days, and study starting at or before peak initiation date), the bias was always below $7 \%$ in absolute value and the estimate was unbiased, on average, over eight simulations. In summary, our simulations highlighted the importance of (1) timing the start of study at or before peak nest initiation date; and (2) reducing the interval between visits (at least when the timing of the first visit is correct). Simulations also revealed that (3) efforts to increase detection probability within each sampling occasion are not as rewarding as increasing the number of sampling occasions within a given time frame. If the study starts after peak initiation date or interval length exceeds onethird of the nesting period, we recommend switching to the "standard" staggered-entry formulation of the model.

\section{Dakotas' Blue-winged Teal example}

The statistically significant effects were: site effects on survival probability, cattle effect on survival probability, and stage effect on both survival and detection probabilities (Table 2). The statistically nonsignificant effect of the Robel index of vegetation was in the direction of lower detection and lower survival probabilities for sites with more vegetation. In the following, we present parameter estimates from the model with only significant effects and with the raised cosine distribution (results from the Gaussian model were qualitatively similar). Daily nest survival was almost 1.0 during the egg-laying stage (Fig. 3). Indeed, no nest was recorded as having failed before the expected start of its incubation period, and only seven nests were recorded as having failed before the visit immediately following the start of incubation. Daily nest survival was greater when cattle were present (effect on the logit scale, $1.00 ; 95 \% \mathrm{CI}$ $=0.372-1.63$; Fig. 3). However, this effect was largely compensated for by a negative effect of being in sites 2 and 4, where the majority of nests had cattle around (Fig. 3, Table 3). Nests located where cattle were absent in sites 2 and 4 had an almost zero probability of succeeding $(95 \% \mathrm{CI}=0.0-8.0 \%)$. A major difference between models with continuous phenology functions and the staggered entry ("standard") model is that point estimates of nest success were higher in the latter (Table 3 ), because the standard approach is based on nests that survived until at least the next sampling occasion, which is a bias toward high survival. Detection probability 


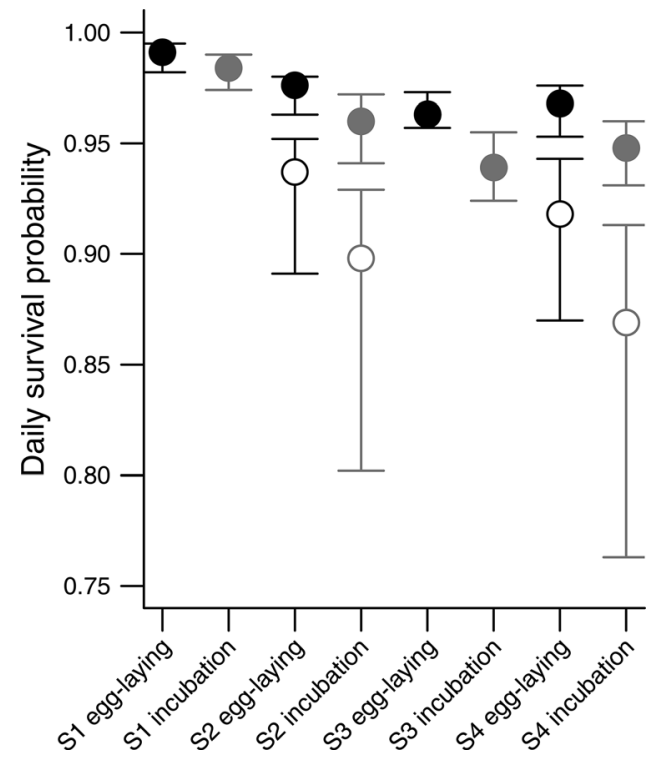

FIG. 3. Daily nest survival probability (mean with $95 \%$ CI) for Blue-winged Teal nests in North and South Dakota study sites. Square shading indicates the stage: black, survival during egg-laying; gray, survival during incubation. For sites 2 and 4 only, solid symbols represent nests in fields with cattle; open symbols are nests in fields without cattle. S1, S2, S3, and S4 designate the four sites. increased from $0.40(95 \% \mathrm{CI}=0.37-0.45)$ during the egg-laying stage to 0.73 during the incubation stage (95\% CI $=0.65-0.81)$. In the standard approach, the probability of not detecting a nest was lowest in site 1 (Table 3, last columns). This difference was not due to detection probability, which did not vary across sites; it was due to survival probability, which was highest in site 1 , so that more nests survived long enough to be subjected to the detection process. Indeed, the estimated probability of nest success was above $60 \%$ in site 1 but around $20 \%$ in the three other sites (Table 3). However, there also was a difference in both the timing of visits and the site-specific phenology, which meant that a greater proportion of nests failed before the start of the study in site 1 (Fig. 4). Breeding density (nest abundance per hectare) varied from 0.61 nests/ha $(95 \% \mathrm{CI}=0.57-$ 0.70 nests/ha) in site 1 to $1.01(95 \% \mathrm{CI}=0.92-1.07$ nests/ ha) in site 3 (Table 3). Site 1 appeared to be the most productive site in terms of successful nests per hectare, but was the least densely occupied of the sites (Table 3).

\section{DisCUSSION}

Our method provides a synthetic framework to estimate the abundance of immobile objects that can be aged upon detection, when detection probability is below 1.0 and objects are submitted to mortality risks in addition to time-to-event processes. Such objects include nests, as in our developed example, but also benthic organisms (using growth marks to age the animals), plants (using life stages), dung piles (using a classifica-

TABLE 3. Estimate of superpopulation size, probability of not detecting a nest, hatching success, and productivity for the four study sites and the three model variants, for Blue-winged Teal nests in North and South Dakota study sites.

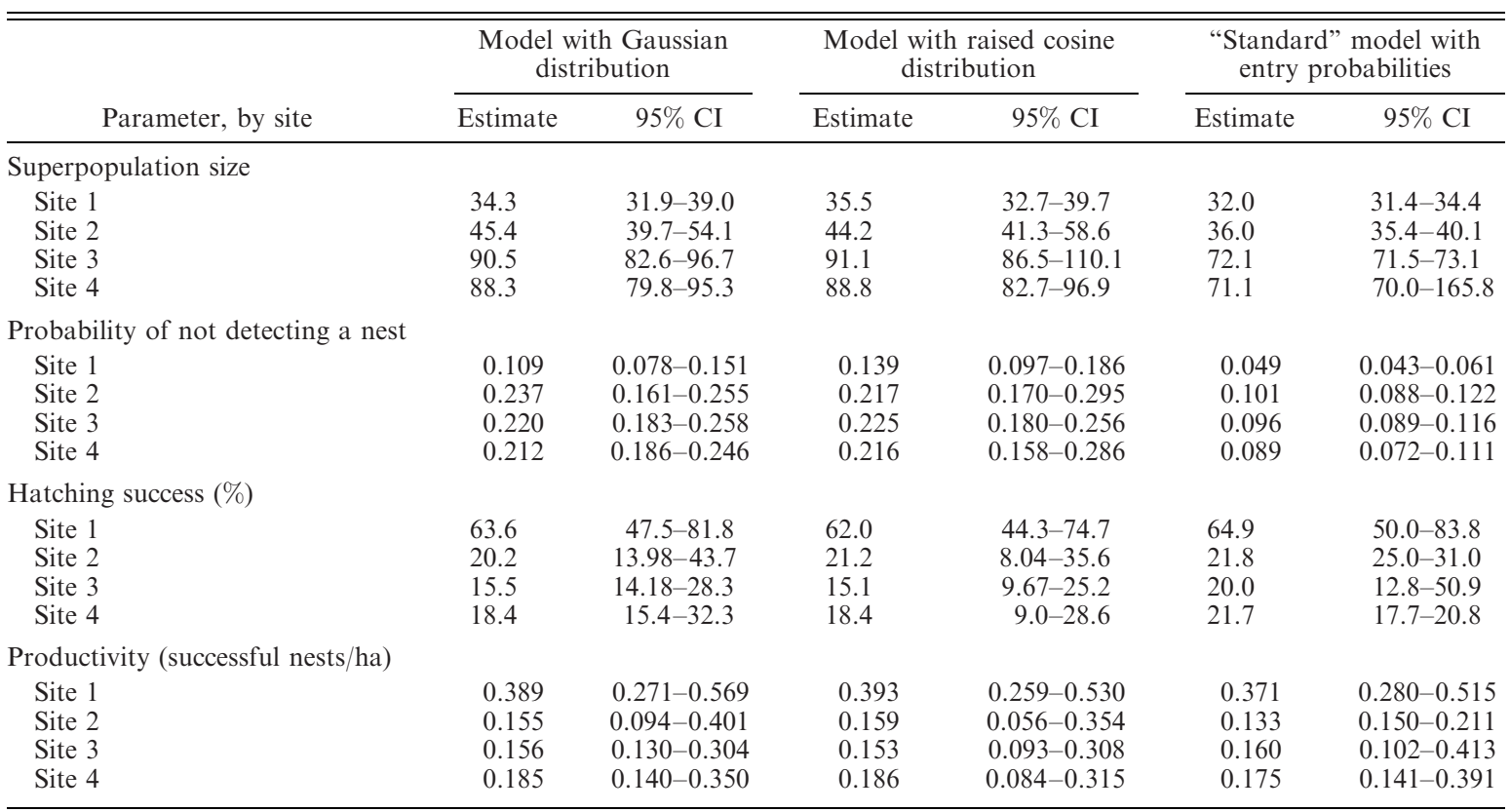

Notes: Models only included significant biological effects. The superpopulation is not defined in the same way for the first two models compared to the third model; hence, the difference in point estimates (see Realistic model variants). Given are point estimates and $95 \%$ confidence interval boundaries from a parametric bootstrap. 

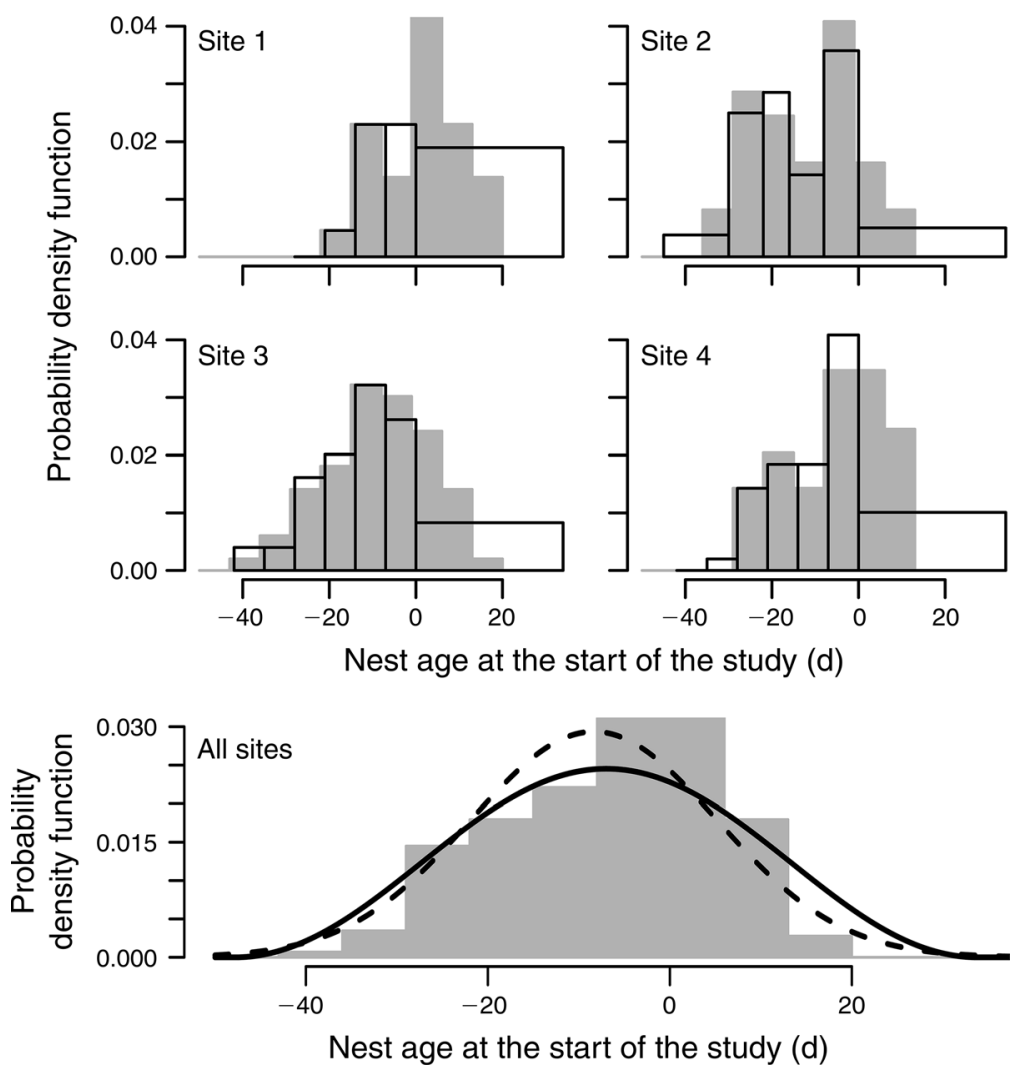

FIG. 4. Density distribution of the age of Blue-winged Teal nests at the start of the study at sites in North and South Dakota. Negative values indicate nests that were initiated after the start of the study. For all panels, the shaded histogram is the distribution among detected nests not accounting for the detection process. The unshaded, black-outlined histogram bars show estimated daily entry rates from the "standard" superpopulation model accounting for the detection process; the rightmost bar spreads over the full potential age range for nests that are present at the first visit to a site (34 days). For the bottom panel (all sites), the solid line is the estimated distribution using the raised cosine model and accounting for the detection process; the dashed line is the estimated distribution using the Gaussian model (neglecting excess kurtosis) and accounting for the detection process.

tion of decay state), and even territorial vertebrates (using growth marks in horns, teeth, shells, and so forth). The method uses data previously intended at estimating only the success of the time-to-event processes, not abundance. In bird population studies, nest success, however, is only one component of reproductive output, so the ability to use these same data to estimate total offspring production for a population or focal area is an important step forward. The key to inference about abundance is information about detection probability. We emphasize that the probability structure for detection (Eq. 3), independently of the structure representing nest initiation dates, represents an innovation because it provides a means for inference about nest abundance. Information about variation in reproductive output is useful for (1) guiding management of bird habitat, (2) monitoring species' response to climate and land use changes, and (3) studying the relationship between habitat choice, as measured by nest abundance, and habitat quality, as measured by breeding success.

Our results can be directly used to compare the relative values of different study sites, i.e., to measure spatially explicit fitness. In our example analysis, we show a twofold variation in breeding productivity (successful nests per hectare) across sites. Nest success was the most import driver of productivity in this system, but without correcting for nest abundance, the between-site variation would have been largely overestimated. Site 1 was both the most successful and the least densely occupied of the four study sites, which also suggests that teal may be unable to predict site-specific breeding success when returning from spring migration, or they may be unable to adapt habitat choice according to anticipated site-specific breeding success. Regarding the effect of nest-site vegetation density on breeding success, our results are statistically nonsignificant, but suggest that nest sites with denser vegetation are less successful, as previously found in steppe-breeding birds (e.g., With 1994), possibly because mammalian nest predators use denser vegetation patches for concealment from raptors.

\section{When should one or the other model variant be used?}

Our application case was, when compared to our simulated scenarios, in the range where estimation is reliable via models with continuous phenology functions 


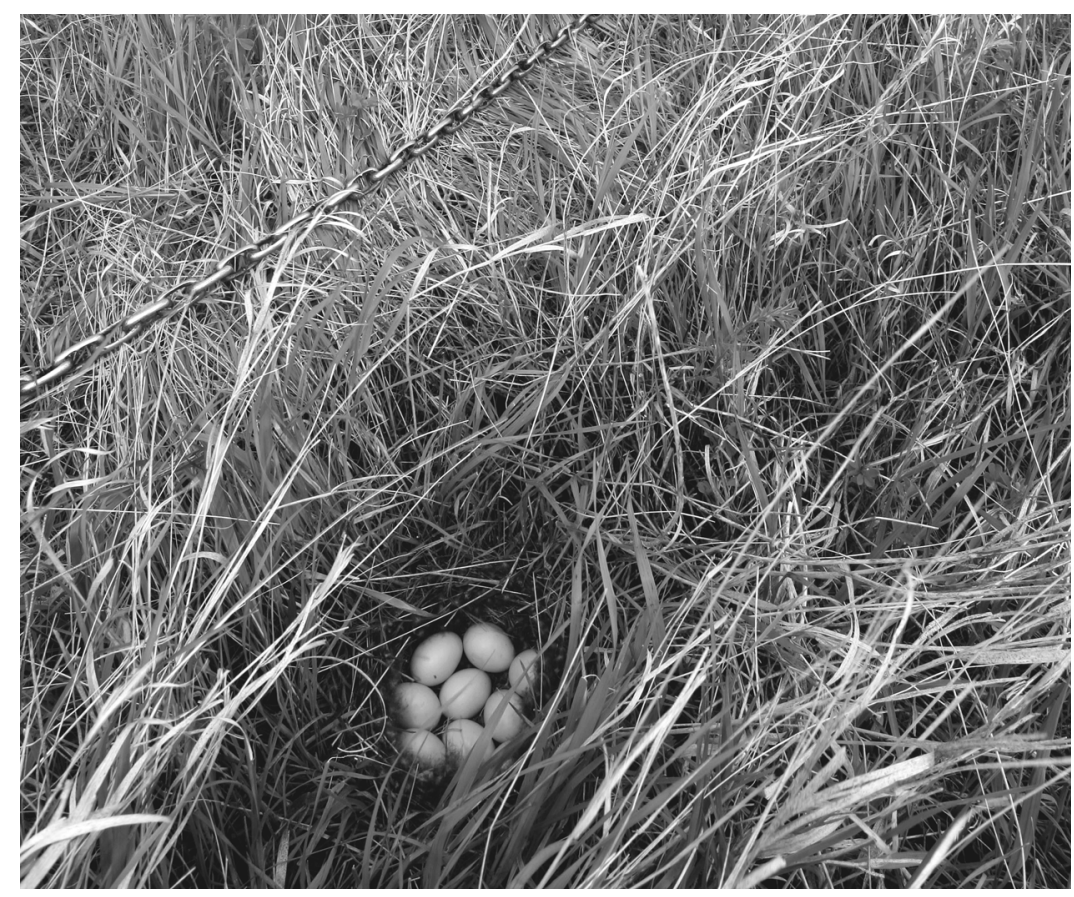

Plate 1. Duck nest found using the chain-drag method at a Dakota (USA) study site. Photo credit: Ducks Unlimited.

(Gaussian or raised cosine); most importantly, peak nest initiation occurred about a week after the start of the study and interval length was about a week; high detection probability also helped to reduce $P_{0}$ below $25 \%$, of which only $5-10 \%$ were nests actually missed by field crews (the rest being never available for detection). As we have highlighted, for sparser data, the continuous distribution models are not well adapted and the staggered-entry approach should be preferred (denoted "TIME" in the software nestAbund). The quantity to be estimated is then the number of nests available during at least one sampling occasion. There are a number of post hoc corrections available to account for nests that are initiated and fail between successive sampling occasions (Schwarz et al. 1993), but no correction is possible for nests that are initiated after the study or long before it starts.

\section{Further model extensions}

An advantage of our model for inference about nest success at the scale of the sampled population is the formal incorporation of both success and detection parameters that can be modeled as functions of habitat covariates, nesting stage, and laying date. If both detection probability and nest success vary according to any of these covariates, then nests with higher detection probabilities could be overrepresented in the sample of detected nests. In most standard approaches to inference about nest success, there is no ability to adjust the estimate of nest success by these different probabilities of nest inclusion in the sample, whereas under our approach this can be done. However, even after accounting for variation associated with identified covariates, it is always possible that substantial heterogeneity remains in either nest success or detection probability. If this heterogeneity is characterized by a covariance between probabilities of detection and nest success (e.g., higher probabilities of success for more concealed nests), then misleading inferences could still result. A possible model extension would be to incorporate either finite mixture (e.g., Norris and Pollock 1996) or random effects approaches (e.g., Natarajan and McCulloch 1999) to deal with (possibly dependent) heterogeneity in probabilities of nest detection and success.

Other potential improvements that we foresee include site specificity in the distribution of nest initiation dates. Indeed, in our teal example we suspect that the breeding season started earlier at site 1, which led to overestimation of $P_{0}$ and $N$ for this site when fitting a model where all sites had the same phenology. Second, the ability to jointly analyze data from multiple species appears to be useful in the context of duck studies in which multiple species co-occur. Third, between-nest variation in $L_{3}$ and $E$ may be introduced using a mixture-model approach with proportion vectors describing the probability that a nest from the superpopulation takes some given $L_{3}$ and $E$ values. Fourth, right-censoring (the fact that the field season may end before some nests have either succeeded or failed) could readily be accommodated by censoring Eq. 1 at the appropriate time.

Last, our model was inspired by duck studies, but can readily be adapted for bird species in which age determination of the unhatched eggs is difficult, but 
the species have altricial young that stay in the nest after hatching (passerines, Columbidae, and so on). In these species, it is often possible to determine the age of the nestlings (e.g., Hanson and Kossack 1963). Then, information about egg age is replaced by information about nestling age; "nest initiation date" is replaced by "clutch completion date"; and the quantity estimated becomes the number of nests that reach the incubation stage rather than the number of nests that are initiated. In other words, inference about detection probability can be based on nests that eventually hatch at least one egg. Implementation of these extensions would be especially useful in increasing the applicability of our approach to the majority of bird species for which unhatched eggs are not easily aged.

\section{ACKNOWLEDGMENTS}

This work was supported by the U.S. Fish and Wildlife Service. We thank three reviewers for their helpful comments.

\section{Literature Cited}

Ackerman, J. T., and C. A. Eagles-Smith. 2010. Accuracy of egg flotation throughout incubation to determine embryo age and incubation day in waterbird nests. Condor 112:438-446.

Calero-Riestra, M., J. T. Garcia, J. Herranz, and F. Suarez. 2013. Breeding output and nest predation patterns in steppeassociated Mediterranean birds: the case of the Tawny Pipit Anthus campestris. Journal of Ornithology 154:289-298.

Collett, D. 2003. Modelling survival data in medical research. Chapman and Hall, London, UK.

Crosbie, S. F., and B. F. J. Manly. 1985. Parsimonious modeling of capture-mark-recapture studies. Biometrics 41: 385-398.

Dinsmore, S. J., G. C. White, and F. L. Knopf. 2002. Advanced techniques for modeling avian nest survival. Ecology 83: 3476-3488.

Gimenez, O., and R. Choquet. 2010. Individual heterogeneity in studies on marked animals using numerical integration: capture-recapture mixed models. Ecology 91:951-957.

Golub, G. H., and J. H. Welsch. 1969. Calculation of Gauss quadrature rules. Mathematics of Computation 23:221-230.

Hanson, H. C., and D. W. Kossack. 1963. The mourning dove in Illinois. Southern Illinois University Press, Carbondale, Illinois, USA.

Jiguet, F., R. D. Gregory, V. Devictor, R. E. Green, P. Vorisek, A. Van Strien, and D. Couvet. 2010. Population trends of European common birds are predicted by characteristics of their climatic niche. Global Change Biology 16:497-505.

Jones, S. L., and G. R. Geupel, editors. 2007. Beyond Mayfield: measurements of nest-survival data. Studies in Avian Biology 34. Cooper Ornithological Society, Camarillo, California, USA.

Kalbfleisch, J. D., and R. L. Prentice. 2002. Failure time models. Pages 31-51 in J. D. Kalbfliesch and R. L. Prentice. The statistical analysis of failure time data. John Wiley, New York, New York, USA.

Kerbiriou, C., I. Le Viol, A. Robert, E. Porcher, F. Gourmelon, and R. Julliard. 2009. Tourism in protected areas can threaten wild populations: from individual response to population viability of the chough Pyrrhocorax. Journal of Applied Ecology 46:657-665.

Klett, A. T., H. F. Duebbert, C. A. Faanes, and K. F. Higgins. 1986. Techniques for studying nest success of ducks in upland habitats in the prairie pothole region. Resource Publication 158. U.S. Department of the Interior, Fish and Wildlife Service, Washington, D.C., USA.

Martin, T. E. 1995. Avian life history evolution in relation to nest sites, nest predation, and food. Ecological Monographs 65:101-127.

Mayfield, H. 1961. Nesting success calculated from exposure. Wilson Bulletin 73: 255-261.

Natarajan, R., and C. E. McCulloch. 1999. Modeling heterogeneity in nest survival data. Biometrics 55:553-559.

Newmark, W. D., and T. R. Stanley. 2011. Habitat fragmentation reduces nest survival in an Afrotropical bird community in a biodiversity hotspot. Proceedings of the National Academy of Sciences USA 108:11488-11493.

Norris, J. L., and K. H. Pollock. 1996. Nonparametric MLE under two closed capture-recapture models with heterogeneity. Biometrics 52:639-649.

Péron, G., P. A. Crochet, P. F. Doherty, and J.-D. Lebreton. 2010. Studying dispersal at the landscape scale: efficient combination of population surveys and capture-recapture data. Ecology 91:3365-3375.

Pollock, K. H., and W. L. Cornelius. 1988. A distribution-free nest survival model. Biometrics 44:397-404.

Ricklefs, R. E. 1969. An analysis of nesting mortality in birds. Smithsonian Contributions to Zoology 9:1-48. Smithsonian Institution Press, Washington, D.C., USA.

Robel, R. I., J. N. Briggs, A. D. Dayton, and L. C. Hulbert. 1970. Relationships between visual obstruction measurements and weight of grassland vegetation. Journal of Range Management 23:295-307.

Robertson, B. A., and R. L. Hutto. 2006. A framework for understanding ecological traps and an evaluation of existing evidence. Ecology 87:1075-1085.

Rotella, J. J., S. J. Dinsmore, and T. L. Shaffer. 2004. Modeling nest-survival data: a comparison of recently developed methods that can be implemented in MARK and SAS. Animal Biodiversity and Conservation 27:187-205.

Schwarz, C. J., and A. N. Arnason. 1996. A general methodology for the analysis of capture-recapture experiments in open populations. Biometrics 52:860-873.

Schwarz, C. J., R. E. Bailey, J. R. Irvine, and F. C. Dalziel. 1993. Estimating salmon spawning escapement using capture-recapture methods. Canadian Journal of Fisheries and Aquatic Sciences 50:1181-1197.

Stanley, H. R., and W. D. Newmark. 2010. Estimating length of avian incubation and nestling stages in Afrotropical forest birds from interval-censored nest records. Auk 127:79-85.

Weller, M. W. 1956. A simple field candler for waterfowl eggs. Journal of Wildlife Management 20:111-113.

Williams, B. K., J. D. Nichols, and M. J. Conroy. 2002. Analysis and management of animal populations. Academic Press, San Diego, California, USA.

With, K. A. 1994. The hazards of nesting near shrubs for a grassland bird, the McCown's Longspur. Condor 96:10091019. 